\title{
Research on the evaluation index system of fashion design
}

\author{
Li Feng ${ }^{1, a}$, Xiaogang Liü, b \\ ${ }^{1}$ College of fashion \& art design, Donghua University, Shanghai 200051, China; \\ afl_dj@dhu.edu.cn, ${ }^{\mathrm{b}}$ liuxg@dhu.edu.cn
}

Keywords: Fashion design, evaluation system, index system.

\begin{abstract}
Scientific evaluation of fashion design is the need of modern design. This research discusses the basic structure of fashion design evaluation system. From the designer's point of view, fashion design elements was divided into core design and extension design, as basic for the establishment of fashion design evaluation system. According to the process of feeling of the wearer, evaluation index system of fashion design was divided into core index system and extension index system, and the evaluation variables with reference value are set up. This design evaluation index system has certain adaptability, and can be flexibly set according to the actual situation.
\end{abstract}

\section{The basic composition of the fashion design evaluation system}

Usually, fashion design contains by six basic elements, according to theirs main bear and weight in design can be divided into two parts of core design and extension design. The core design is composed of three main factors, including styling design, color design and fabric design. Extension design is composed of other three main factors, structure design, technology design and decoration design. Therefore, the evaluation of fashion design is mainly aimed at these six major elements of fashion design, the evaluation system is shown in figure 1.The core design part consists of three subsystems, including styling design evaluation subsystem, color design evaluation subsystem and fabric design evaluation subsystem. The extension design part includes three subsystems, which are structure design evaluation subsystem, technology design evaluation subsystem and decoration design evaluation subsystem.

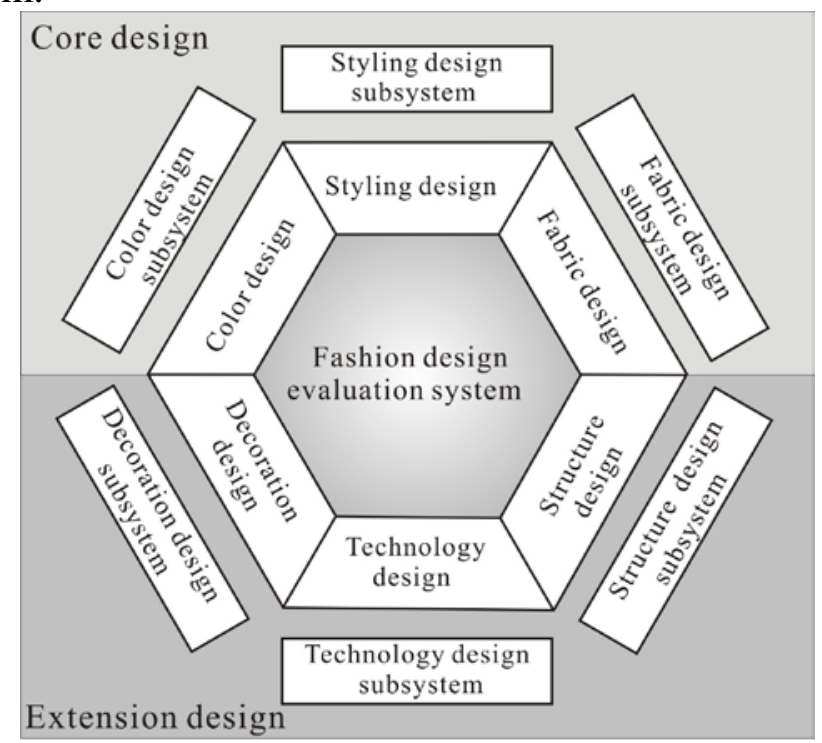

Fig. 1 The basic composition of the fashion design evaluation system

\section{The formation process of the index system of fashion design evaluation}

In the fashion design evaluation system, the first step is to determine the design evaluation index. Determination and selection of evaluation index directly determine the direction of evaluation and the emphasis of evaluation results. Therefore, in the entire fashion design evaluation system, must construct the appropriate design evaluation index system. 
Evaluation index means that evaluation object a characteristic concept and quantity, it is clearly a evaluation object a characteristic concept (i.e., properties, and a reflection of the number of evaluation objects, with qualitative and quantitative understanding of the dual role. According to the needs of the evaluation of characters and goals, it is able to comprehensively and systematically reflect a series of relatively complete and mutually organic links of evaluation index system ${ }^{[1]}$. Evaluation index and evaluation index system are the true reflection of whole or part of the evaluation object, and the accurate reflection of true degree of thing is the basic guarantee for the accurate and reliable conclusion of scientific evaluation.

Evaluation index includes index name and scalar quantity, both of them must be determined according to the specific circumstances. On the one hand, evaluation index is based on the evaluation information, such as relevant information of evaluation object, including design information, material information, business information and so on. This information determines which properties of the evaluation object, these attributes are objective existence, when the design is completed, no change. On the other hand, evaluation index is determined by the purpose and objectives of evaluation. Evaluation purpose and objectives are differently, the selection of evaluation index is different.

Not all attributes of evaluation object will enter evaluation system to become the evaluation index. There must be some difficulty in evaluating all the attributes of the evaluation object, and evaluation index should be selected according to the purpose of evaluation. Evaluation screening generally follow the scientific and practical, systematic and obtain, completeness and exclusive, accuracy and simplicity, timeliness and versatility, effective and specific, quantitative index and qualitative index, absolute index and relative index, static index and dynamic indexes of the dialectical unity of the main principles.

The formation process of evaluation index system includes five steps: preparation stage, the formation of general index system, index system primary election, index system monitoring, index system practical application and establishment ${ }^{[2]}$, see figure 2.

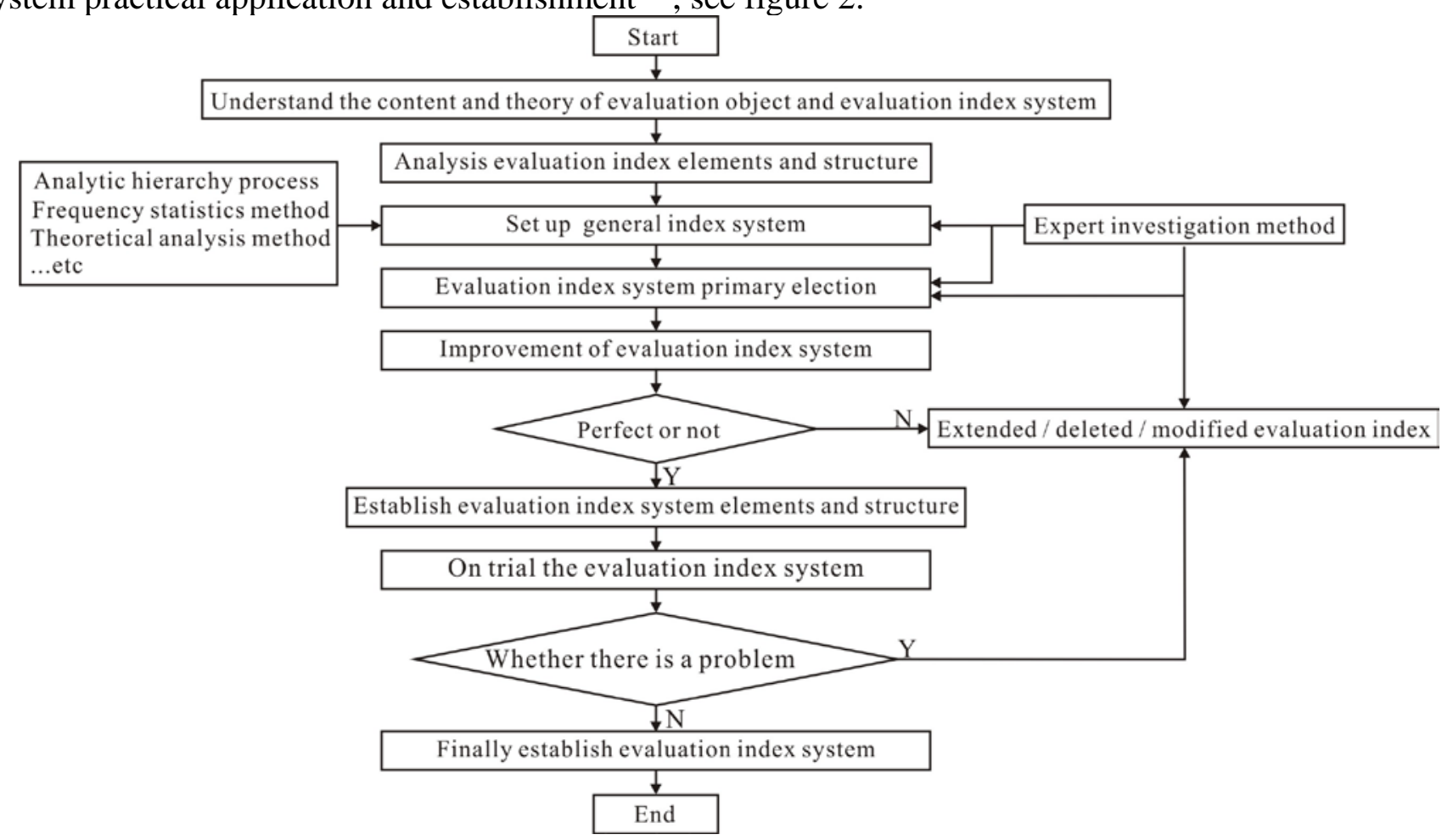

Fig. 2 The formation process of evaluation index system

Evaluation object of fashion design evaluation has multiple attributes, and it should give the evaluation of comprehensive value of the object. Therefore, fashion design evaluation is multi index comprehensive evaluation. Completion of the fashion design evaluation mainly to solve the following five aspects basic problems:

1. Determine evaluation index system. Including evaluation index design and evaluation index select. 
2. Determine the scale of evaluation (that is, the standard). Used to determine the evaluation object and determine its value, to assign each evaluation index.

3. Processing evaluation index. Turn the actual value of index into index value.

4. Determine the weight coefficient of each evaluation index. The common methods are divided into subjective weighting method and objective weighting method. Subjective weighting method including: Delphi method, dualistic comparison method and AHP. Objective weighting method includes: the reciprocal of the variance, the coefficient of variation, the reciprocal of the negative correlation coefficient, the right of the right of business, etc.. There is also a combination of the combination weighting method ${ }^{[3]}$.

5. Select evaluation method. Fashion design evaluation is a multi index comprehensive evaluation, multi index comprehensive evaluation method includes: qualitative analysis, quantitative analysis, comprehensive analysis method.

According to the above principles, research constructed fashion design evaluation system, including main system and subsystem.

\section{Composition of fashion design evaluation index system}

According to the basic structure of fashion design evaluation system by figure 1, from the point of view of designer, fashion design evaluation index system can be divided into two main system, respectively, core index system of fashion design evaluation and extension index system of fashion design evaluation.

\subsection{Core index system of fashion design evaluation}

Styling, color and fabric are the most important elements of fashion designers carry out in design work, so the core index system of fashion design evaluation includes styling design evaluation subsystem, color design evaluation subsystem and fabric design evaluation subsystem, as shown in figure 3.

Styling evaluate mainly for the evaluation of external styling and internal styling. So, set silhouette, proportion and profile modeling as evaluation variable for external styling. Set collar, shoulders, sleeves, placket, waist and hem as evaluation variables for internal styling.

The basic attribute of color is used as the index of color evaluation, including hue, purity, brightness and tone. Each index is set to the relevant evaluation variables. Such as cool, worm, low medium, high, contrast, coordinate etc..

The sense of fabric is the basic of fabric evaluate, the order of perception is look, touch, feel. In the look stage, fabric quality and style are the evaluation variables. In touch stage, make soft and hard, thickness, weight for evaluation variables. Feel refers to the feelings after wearing of clothing, there set smooth and elastic as evaluation variables.

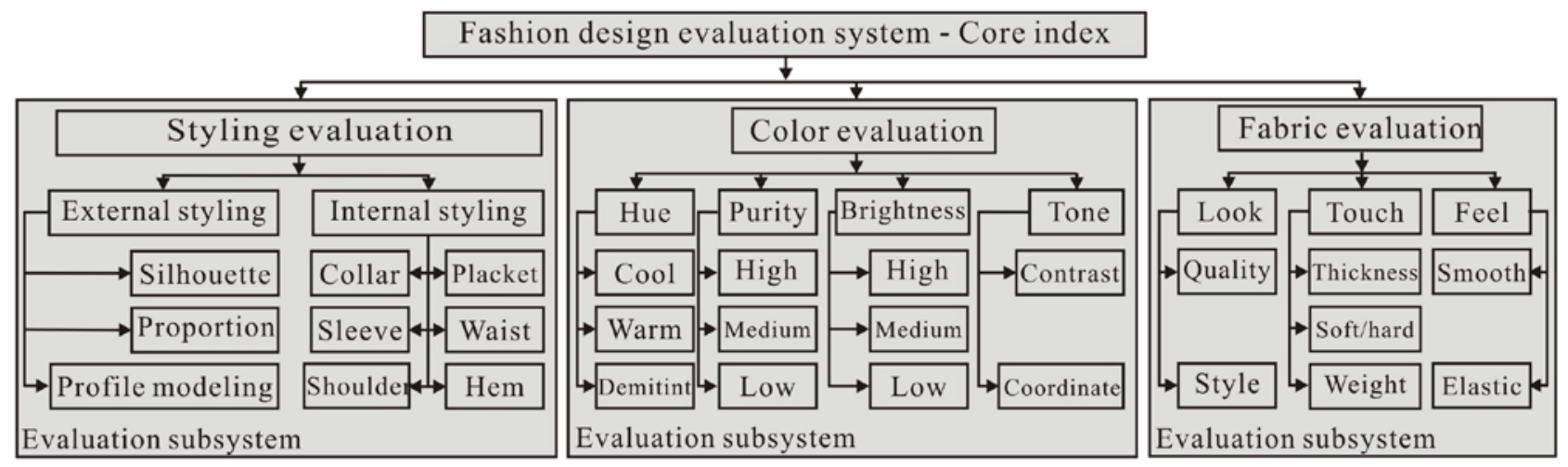

Fig.3 Core index system of fashion design evaluation

\subsection{Extension index system of fashion design evaluation}

When the evaluation object is garment or sample, the importance of structure design and technology design is almost equivalent to the styling, color and fabric design. When the evaluation object is design manuscript, the show effect of structure, technology and decoration design is weaker 
than styling, color and fabric design. The effectiveness, accuracy and availability of evaluation are relatively weak.

Therefore, the extension index system of fashion design evaluation includes structural design evaluation subsystem, technology design evaluation subsystem and decoration design evaluation subsystem, see Fig.4.

Structural evaluation with clothing functional, decorative and fitness as the evaluation index hierarchy. These three indexes are all set cut line, pleat and dart for evaluation variables.

Technology evaluation mainly set integral, component and special technology as hierarchical index. They all used stitching, line and ironing as evaluation variables.

Decorative evaluation contains a lot of sub level index, such as mosaic, pattern, destruction, embroidery, zipper, button, etc.. According to the evaluation object decoration method is adopted as the index setting. The setting of evaluation variables should be carried out according to characteristics of decorative techniques.

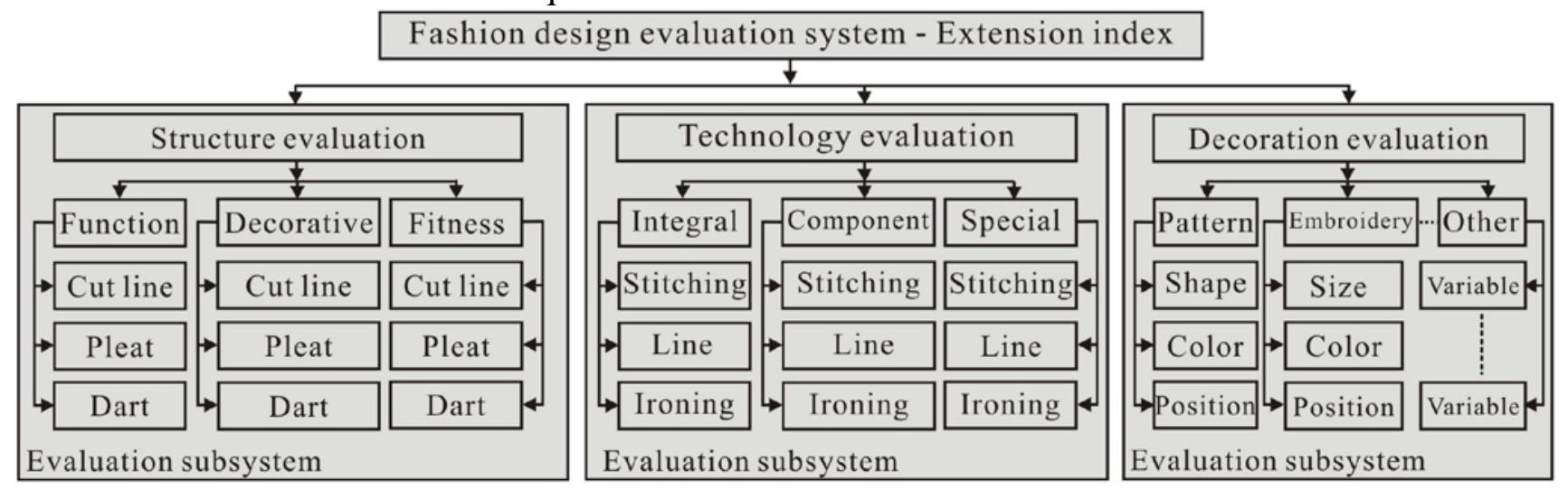

Fig.4 Extension index system of fashion design evaluation

\section{Summary}

In this research, fashion design evaluation index system is divided into two modules, which are relatively independent and symmetrical, in order to improve efficiency, enhance comparative in the evaluation of organization and data collection, data analysis, comprehensive evaluation of the work. The index can also have other development. If the above six design elements are listed as the core design index, can also add functional design or pattern design or others as extension design index. In addition, the index system of fashion design evaluation could add and delete subsystem according to the actual needs, evaluation variables setting also according to the actual situation.

\section{References}

[1] Lou Cequn, The theory and method of the measurement of the social science evaluation, Huazhong Normal University press, Wuhan, 1999,103

[2]Qiu Junping, Wen Tinxiao, etc., Evaluation study: theory, method and practice, Science press, Beijing, 2010,139

[3] Hu Yonghong, He Sihui, Comprehensive evaluation method, Science Press, Beijing, 2000, 78 